\title{
A Multi-Frequency SDR-Based GBSAR: System Overview and First Results
}

\author{
Adrià Amézaga ${ }^{1,2, *} \mathbb{B}$, Carlos López-Martínez ${ }^{1} \mathbb{1}$ and Roger Jové ${ }^{2}$ \\ 1 Remote Sensing Lab (RSLab), Department of Signal Theory and Communications (TSC), Campus Nord, \\ Universitat Politècnica de Catalunya-BarcelonaTech (UPC), Jordi Girona 1-3, D4 Building, \\ 08034 Barcelona, Spain; carlos.lopezmartinez@upc.edu \\ 2 Balam Ingeniería de Sistemas SL, 08792 La Granada, Spain; roger.jove@balamis.com \\ * Correspondence: adria.amezaga@upc.edu
}

check for updates

Citation: Amézaga, A.;

López-Martínez, C.; Jové, R. A

Multi-Frequency SDR-Based GBSAR System Overview and First Results.

Remote Sens. 2021, 13, 1613.

https://doi.org/10.3390/rs13091613

Academic Editor: Isaac Ramos

Received: 17 March 2021

Accepted: 18 April 2021

Published: 21 April 2021

Publisher's Note: MDPI stays neutral with regard to jurisdictional claims in published maps and institutional affiliations.

Copyright: (c) 2021 by the authors. Licensee MDPI, Basel, Switzerland. This article is an open access article distributed under the terms and conditions of the Creative Commons Attribution (CC BY) license (https:/ / creativecommons. org/licenses/by/4.0/).

\begin{abstract}
This work describes a system-level overview of a multi-frequency GBSAR built around a high performance software defined radio (SDR). The main goal of the instrument is to be employed as a demonstrator and experimental platform for multi-frequency GBSAR campaigns. The system is capable of operating in $\mathrm{P}, \mathrm{L}, \mathrm{C}$ and X-bands, and signal generation and digital signal processing are customizable and reconfigurable through software. An overview of the software and hardware and implementations of the system are presented. The operation of the system is demonstrated with two measuring campaigns showing focused amplitude images at different frequencies. It is shown how the usage of SDR for GBSAR systems is a viable design option.
\end{abstract}

Keywords: software defined radar; GBSAR; software defined radio; synthetic aperture radar; multifrequency radar

\section{Introduction}

Software defined radar (SDRadar) has gained attention in the past few years. SDRadar is a relatively new paradigm that arose from the natural evolution of radar system design. Early radars relied fully on analog hardware, including data presentation to the user. Throughout history, advances in electronic technology have allowed replacing analog parts of the system with digital ones. Digital processing allows high flexibility and robustness, while easing design by eliminating analog design difficulties. The natural evolution has been to replace parts that are later in the analog chain, beginning with data presentation and recording, and continuing to base-band and intermediate-frequency (IF) processing. As digital processing capabilities increase, more and more parts are being replaced by digital ones. SDRadar arises from this natural evolution and refers to the usage of software for implementing many processing operations formerly done with specialized analog or fixed digital hardware.

SDRadars are usually implemented using software defined radios (SDR). The concept of SDR is analogous to SDRadar, but more general since it refers to radios in general. SDRs in the market target mainly communication applications. Since the communications market is massive, SDRs have benefited from intensive investment and research and are nowadays highly integrated and robust. It has to be noted that the benefits of using a SDR for this task manifest themselves in the designer side rather than in the user one. In general, the benefits are related to the increased design flexibility and significantly reduced design cycles.

According to the Institute of Electrical and Electronic Engineers (IEEE), an SDR is a "radio in which some or all the physical layer functions are implemented in software" [1]. IEEE clarifies this definition by stating that "a radio can be an SDR as long as the characteristics of the transmitted signal can be modified after manufacture through software and/or firmware downloads [1]". 
One of the first authors to talk about SDRadar was Wiesbeck [2]. In his paper, Wiesbeck proposed a new kind of synthetic aperture radar (SAR) that is software-defined, to reduce weight, increase its reliability and ease its calibration. Later publications focused on the design of radar prototypes built around commercial SDRs or digital processing blocks. The majority of them used the Universal Software Radio Peripheral (USRP) [3-7]. Many applications of SDRadar have been described, including, but not limited to: passive radar using signals of opportunity [8,9], synthetic aperture radar [10-12], hybrid radar and communication systems [4,10], ground penetrating radar [13], MIMO Radar [14,15] and cognitive radar [16-18].

This work is centered on the use of SDRs for a multi-frequency software defined ground based synthetic aperture radar (GBSAR). A GBSAR is a popular remote sensing system used in the local monitoring of landslides, civil infrastructure, mines, buildings, glaciers and other geological and physical phenomena $[19,20]$. A GBSAR exploits the synthetic aperture technique, a tool capable of generating high resolution images of the complex electromagnetic reflectivity of a target scene. GBSARs are complimentary to space-borne or air-borne SAR systems. The advantages of GBSARs over airborne or space-borne systems are: continuous and time-critical monitoring, ease of deployment, favorable geometrical configuration of the measurement in some situations, simpler data processing and lower cost. Their main disadvantage is their limitation to the imaging of much smaller areas.

Early GBSARs were based on vector network analyzers (VNA). These systems relied on VNAs to perform linear frequency sweeping and coherent signal recording. The VNA provided flexibility and a ready-to-use device to implement the radar core. However, those systems were bulky, extremely expensive due to the high cost of the VNA and suffered from a slow scanning time. Due to these drawbacks, GBSAR manufacturers gradually replaced VNAs for custom electronic designs that allowed custom functionalities and reductions in the scanning time by one order of magnitude (from few minutes to less than a minute) [20], at the expense of flexibility. GBSAR systems can benefit from the SDRadar paradigm by gaining operational flexibility, integration and robustness, and having low costs.

Radar frequency plays an important role in the measurement. High frequencies lead to increased azimuth resolution and smaller systems. However, systems that operate at high frequencies are more sensitive to scene decorrelation effects, and suffer from increased free-space loss. On the other hand, low-frequency operation leads to bigger antennas, less resolution and less sensitivity to decorrelation effects [19]. It has also been shown that high frequencies are more affected by atmospheric conditions such as turbulence and humidity gradients [20-22].

It has been shown that frequency diversity has significant benefits [23-25]. A notable advantage is the capability of exploiting the response of the target to each frequency. Certain geophysical parameters of a target interact in different ways depending on frequency. Often, a certain frequency band is more suitable than other to extract those parameters. For example, snow mapping is better done at C-band [26,27]. The sensing of surfaces with vegetation cover is also possible only at low-frequency bands, such as L-band and $\mathrm{P}$-band, due to its high penetration regarding vegetation. The combination of two or more frequency bands when sensing the same target has also found other applications, such as biomass parameter extraction [28].

The instrument described in this work is a multi-frequency GBSAR built around a highperformance SDR. The main goal of designing this instrument was for it to be employed as a demonstrator and experimental platform for multifrequency GBSAR campaigns. The system is capable of operating in $\mathrm{P}, \mathrm{L}, \mathrm{C}$ and $\mathrm{X}$-bands. Section 2 details the software and hardware design and an implementation of the system; and data system control and processing aspects. Section 3 describes some of the measurement campaigns and their results. Finally, Section 4 summarizes the main conclusions of this work. 


\section{System Description}

This section aims at providing a high level description of the developed system. The system-level aspects are first described, followed by a description of its hardware and software parts.

\subsection{System Requirements}

The GBSAR system to be developed has to comply with certain performance requirements that depend, basically, on the intended application and on the target to be measured. In order to get a sense of an acceptable performance, two popular GBSARs in the industry are considered as references. The first one is the IBIS-FM from Ingegneria dei Sistemi (IDS) [29], and the second one is the FastGBSAR-S from Metasensing [30]. Their most important characteristics are summarized in Table 1 . Note that the parameters are very similar, with a notable disparity in the scan time and transmit power. These two parameters are directly related, as will be shown.

Table 1. Comparison of two popular GBSARs in the market.

\begin{tabular}{lrr}
\hline Parameter & IBIS-FM & FastGBSAR-S \\
\hline Frequency & $17.2 \mathrm{GHz}$ & $17.2 \mathrm{GHz}$ \\
Maximum Range & $4.5 \mathrm{~km}$ & $4 \mathrm{~km}$ \\
Displacement accuracy & $0.1 \mathrm{~mm}$ & $0.1 \mathrm{~mm}$ \\
Maximum TX Power & $12 \mathrm{dBm}$ & $42 \mathrm{dBm}$ \\
Range resolution & $0.5 \mathrm{~m}$ & $0.5 \mathrm{~m}$ \\
Azimuth resolution & $4.8 \mathrm{rad}$ & $4.3 \mathrm{rad}$ \\
Minimum scan time & $<3 \mathrm{~min}$ & $>5 \mathrm{~s}$ \\
\hline *SNR $>$ 20dB. Does not include atmospheric effects. & &
\end{tabular}

Although not specified by the manufacturers, it is considered that the worst accuracy occurs at maximum range. Considering these parameters, and after considering various trade-offs such as cost, hardware complexity and maximum range, the design goals of our system can be established as seen in Table 2 .

Table 2. Design goals.

\begin{tabular}{lc}
\hline Bands & $\mathrm{P}, \mathrm{L}, \mathrm{C}, \mathrm{X}$ \\
Maximum range & $2 \mathrm{~km}$ \\
Displacement accuracy & $<1 \mathrm{~mm}$ \\
Acquisition time per image & $<10 \mathrm{~s}$ \\
Range resolution & $<1 \mathrm{~m}$ \\
Aperture length & $2 \mathrm{~m}$ \\
\hline
\end{tabular}

Note that the maximum range and displacement accuracy have been relaxed with respect to the two systems shown in Table 1 . This was done to limit the required transmission power, which reduces significantly the cost of the er, while keeping the scan time in the order of seconds. The accuracy and range values are considered acceptable for a prototype and the intended measurements.

A parameter necessary for the maximum range calculation is the scattering coefficient $\left(\sigma_{0}\right)$, which was set to $-25 \mathrm{~dB}$, a value common for relatively smooth surfaces at grazing angles around $30^{\circ}$ [31]. Using this relation and the other design parameters, the SNR budget determined that a $10 \mathrm{~s}$ acquisition time was sufficient.

\subsection{Hardware Overview}

The implemented GBSAR system consists of a coherent transmit-receive unit mounted on a linear rail. The radar core includes a software defined radio (SDR) and an external front-end employed for frequency extension, filtering, frequency modulated continuous wave (FMCW) deramping and transmit-receive leakage mitigation. The linear unit consists 
of a robust steel rail and a control system comprising a servomotor, a motor controller and an Ethernet switch that provides a single interface for both the SDR and the motor controller. An external host computer commands all movements and all radar unit operations. It also stores the received data for further processing and image generation. Figure 1 shows a high level diagram of the system.

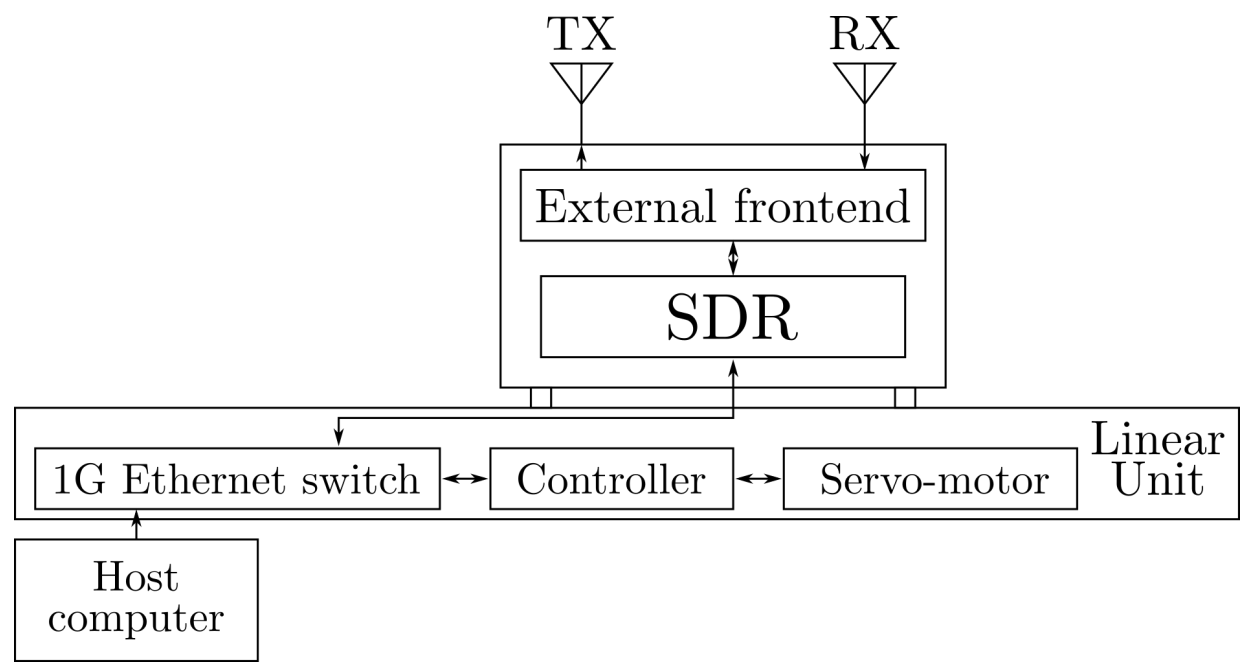

Figure 1. High level diagram of the developed GBSAR system.

\subsubsection{SDR}

The SDR is the core of the system. It supplies signal generation, sampling, digital processing and interfacing with other devices. It has to be noted that the concept of SDR may be ambiguous in some instance due to the diffuse frontier that determines the necessary amount of physical software functions required to catalog a system as an SDR. Figure 2 shows the processing chains implemented in the SDR. The transmitted signal is generated in software and loaded in a random access memory (RAM) block in the FPGA for full sampling rate transmission. On the receiver side, the signal is digitally filtered and decimated before being sent to the host computer for further processing.

When choosing an SDR from the available options, various aspects such as price, number of channels, processing power, transmission and receiving sampling rates and hardware interfaces, among others, play determinant roles. The most determinant aspects that conditioned the decision were the data interface throughput, a flexible processing system with enough room for experimentation and open source code.

A product that fits these requirements is the USRP X310 SDR from Ettus Research. The reasons to use this device were its powerful field programmable gate array (FPGA), its allowing experimentation and its ability to interchange the analog front-ends.

The internal SDR RF front-ends, commonly called daughter-boards, play an important role in the overall performance. The UBX-160 daughter-board from Ettus Research was employed. The UBX-160 board is a Zero-IF transceiver with complete RF front-ends providing carrier frequencies up to $6 \mathrm{GHz}$, with an RF bandwidth of $160 \mathrm{MHz}$. 


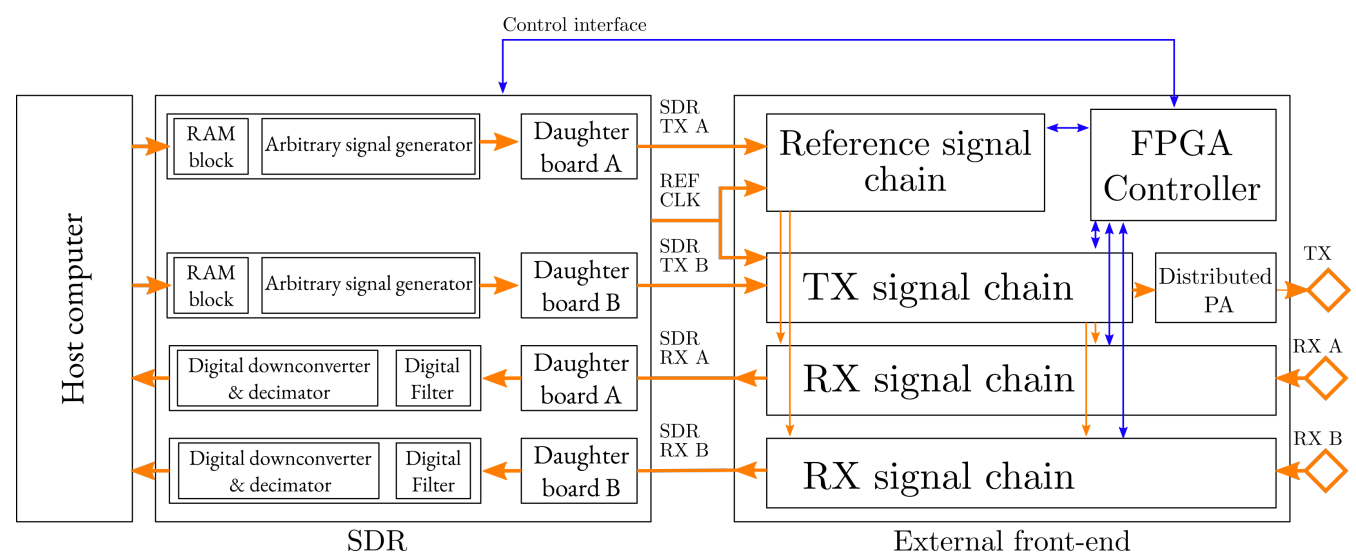

Figure 2. Simplified diagram of the system. Blue arrows are low speed digital signals and orange arrows represent RF signals.

\subsubsection{RF Front-End}

In order to extend the capabilities of the UBX-160 daughter-boards and implement FMCW analogue deramping, an external front-end has been implemented. It was designed with the requirements stated in Section 2.1, and with a special emphasis on flexibility. Note all analog processing could be implemented in a single front-end, and the daughter-boards may be removed in the future. A simplified diagram is shown in Figure 2. The roles of the front-end are:

1. Provide frequency extension to X-band.

2. Amplify the transmit and receive signals.

3. Implement analog FMCW.

4. Reduce the transmit-receive leakage.

5. Limit the signal power to avoid damaging the SDR ports.

The front-end assembly consists of a transmit chain, a reference signal chain and two receive chains to allow simultaneous receiving capability for dual polarization or dual baseline interferometry. Nevertheless, due to practical issues, this publication deals only with VV polarization. The front-end is designed to operate in P, L, C and X-bands and provides a mitigation scheme for transmit-receive leakage inspired by Edwards [32]. Both receive chains are identical; and the transmit and reference chains are similar, differing in the presence of a distributed power amplifier covering frequencies from $\mathrm{DC}$ to $12 \mathrm{GHz}$, and the transmit signal sampling for calibration. It also includes a self observation path to track changes in system gain and phase. For coherent operation, all tones used for up-conversion are derived from the same reference from the USRP X310.

The transmit chain consists of a broadband up-conversion mixer, a frequency synthesized, a switched filter bank and broadband signal drivers, that feed the power amplifier. A resistive coupler samples the transmit signal used to feed the deramping mixers and to use it in system calibration procedures. The receive chain consists of a broadband LNA, deramping mixers, signal drivers and power limiters to avoid damaging the SDR.

The configuration state of the front-end is controlled via the USRP Hardware Driver (UHD) through the General Purpose Input Output (GPIO) signals connector in the USRP X310. A small FPGA receives commands from the SDR and is used mainly as an InputOutput (IO) expander to control the state of RF switches and the power state of components. The switches are used to implement filter banks, bypassing certain components or selecting specific RF sub-chains for each frequency configuration.

\subsubsection{Antennas}

The antennas of a GBSAR system play an important role in determining the area coverage and the maximum range that can be imaged. 
Two antennas were selected. The first one covers the L, C and X-bands, and consists of a wide-band Vivaldi tapered slot aluminum antenna and can be seen in Figure 3a. It covers the full range between $675 \mathrm{MHz}$ and $11 \mathrm{GHz}$. The second antenna, which covers the P-band and is implemented on a PCB substrate, is a log-periodic antenna covering the $400 \mathrm{MHz}-1000 \mathrm{MHz}$ range. This antenna is shown in Figure $3 \mathrm{~b}$.

Both antennas were selected for its low cost and readily available anechoic chamber polar test data. The polar gain plots of both antennas are shown in Figure 4. If the antennas are operated in a vertical polarization configuration, the $\phi=90$ cut corresponds to the gain over azimuth.

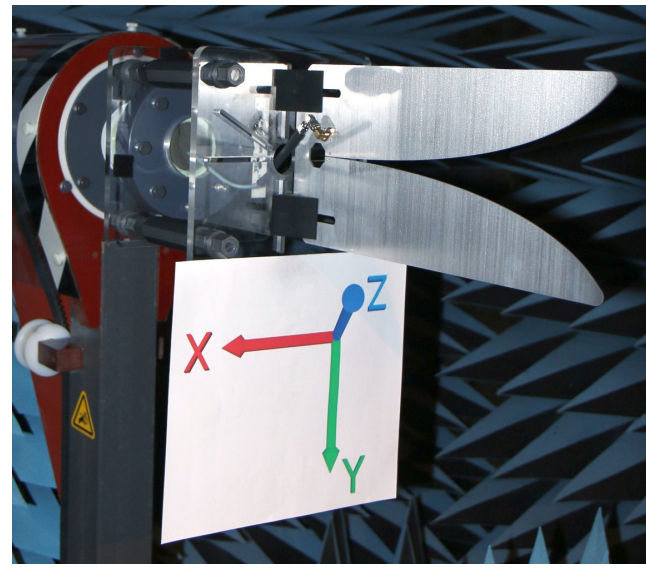

(a) Vivaldi

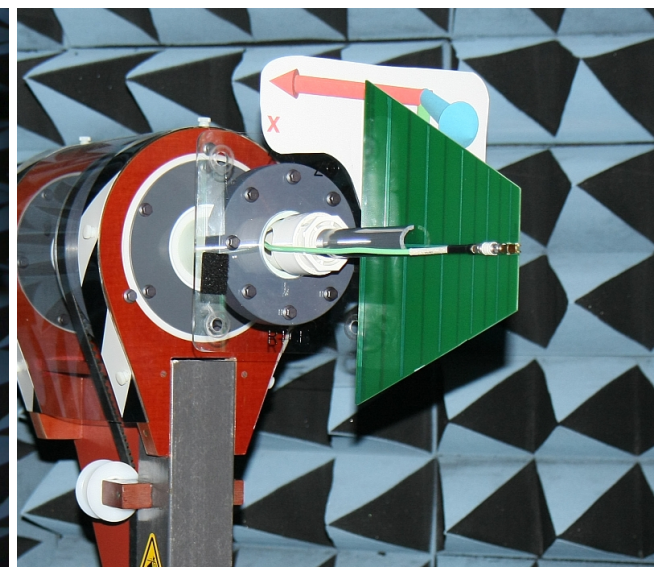

(b) LPDA

Figure 3. Antenna models. Credit: Antenna Test Lab Co.

\subsection{Zero-IF Impairments' Effects on the Radar Signal}

The non-ideal effects of the Zero-IF architectures, present in the majority of SDR front-ends, cannot be ignored when considered in a radar, especially in an FMCW system. The unbalancing effects of the Zero-IF architecture are the origins of two spurs: the carrier leakage and the image frequency [33]. Typically, this imbalance can be calibrated at a certain frequency. However, classical calibration methods are narrow-band, and any departure from the calibration frequency is accompanied by a degradation in spur cancellation. There are wide-band methods that compensate the imbalances in a wider range of frequencies, but require more complicated calibration procedures.

The signal at the RX port is a sum of the signal scattered to the scene and the direct leakage signal from TX. The leakage signal spurs, after deramping, are band-limited frequency sweeps and can mask the much weaker scattered signal.

As can be seen in Figure 5b, the degradation of the noise floor is notable. The interference power cannot be reduced by coherent averaging, such as white Gaussian noise (WGN), because the interference is coherent along sweeps. A possible alternative to reduce the interference power would be to use a sweep spanning only a portion of the upper or lower side-band. This option has the drawback of reducing the range resolution. In order to avoid the degradation in range resolution, an alternative method that preserves the bandwidth has been implemented. This method consists of a model-based extrapolation of the beat frequencies in the STFT domain. The method description and implementation is presented in Neemat [34]. The spur seen in Figure 5a is zeroed and the missing data segment is filled with interpolated data, following a model of the FMCW radar signal. An advantage of this method is that the spur is eliminated completely while avoiding the side-lobe level degradation that would appear by only blanking the spur. 


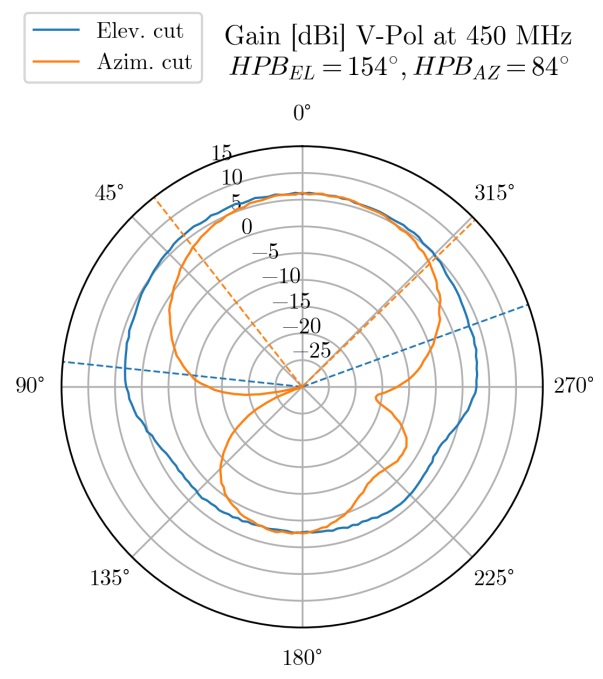

(a) $450 \mathrm{MHz}$

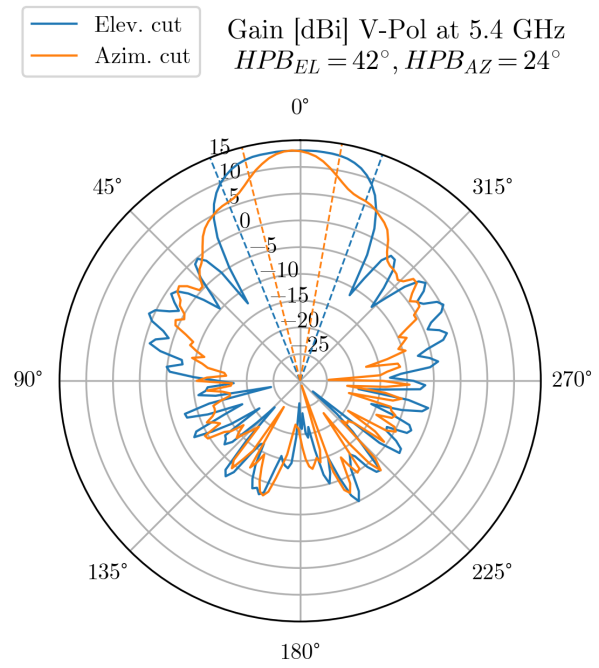

(c) $5.4 \mathrm{GHz}$

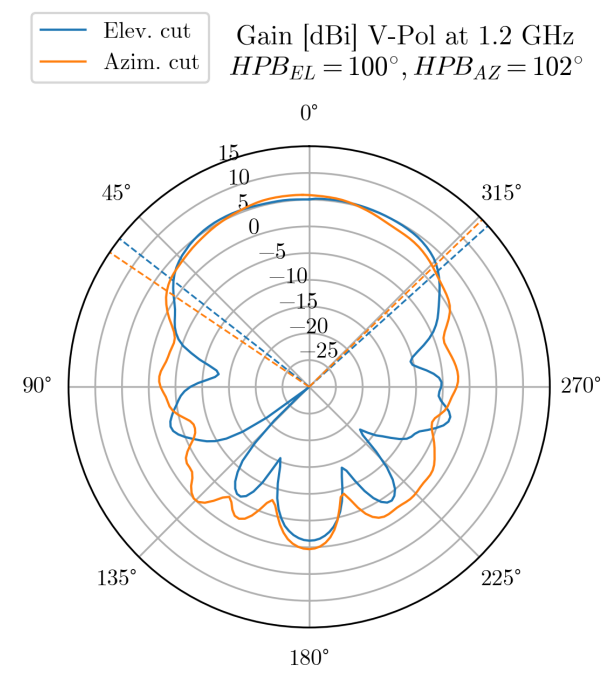

(b) $1.2 \mathrm{GHz}$
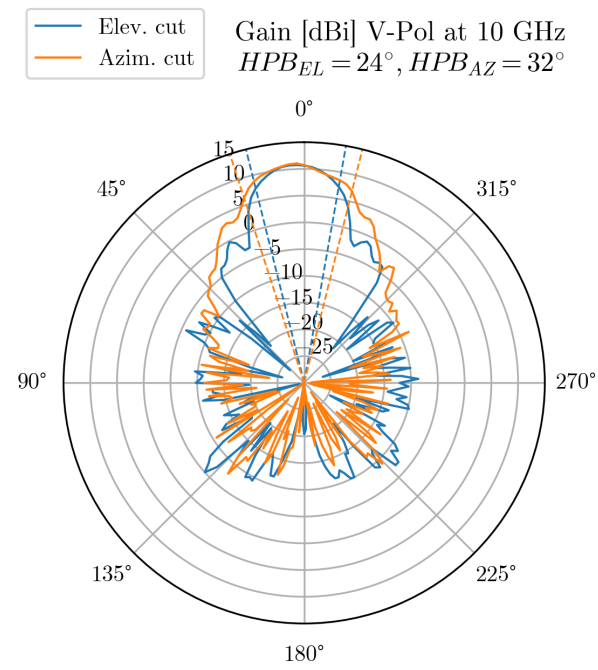

(d) $10 \mathrm{GHz}$

Figure 4. (a) LPDA antenna gain. (b-d) Vivaldi antenna gain. Credits: Antenna Test Lab Co.

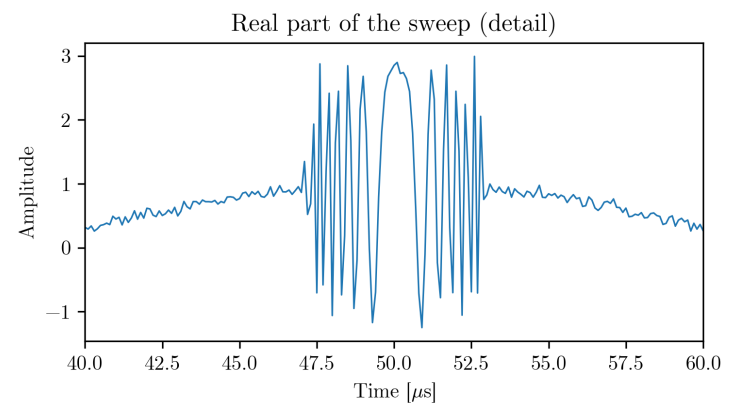

(a) Simulated interference in time domain (detail).

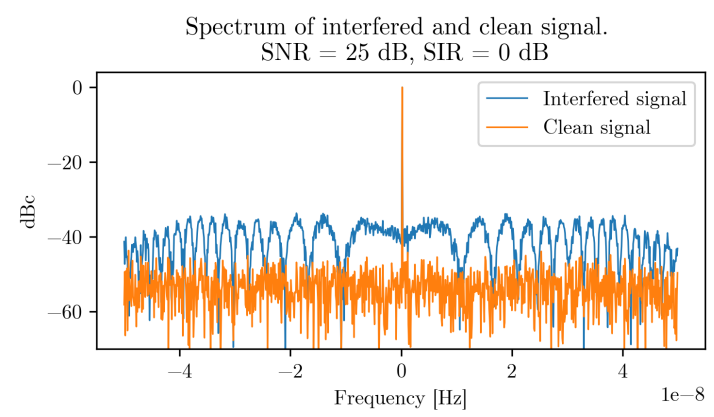

(b) Simulated interfered signal and clean signal in frequency domain.

Figure 5. Time and frequency domain views of the simulated interference. 


\subsection{Digital Implementation}

The high level implementation of the digital radar core can be seen in Figure 6 and is implemented on top of an RF Network-on-Chip (RFNoC) architecture in the SDR FPGA. The radar core consists of two sweep generators implemented via RAM block signal players, which are programmed by the host. The transmitted signal is generated at full rate and sent directly to the radio core block, which is in charge of signal transmission and reception to and from the SDR daughter-boards. The deramped signal is sampled at full rate and is filtered and decimated by a DDC block. The output of the DDC block is connected to a stream endpoint which sends the data to the host computer.

The signal generation scheme implemented in this design is one of the innovations with respect to previously SDRadars reported in the literature (a notable exception is the paper by Prager [35], which presented the same idea at the same time it was being implemented in the present work). The reason for generating the signal inside the FPGA instead of generating it in the host computer, as done in most cases in SDR systems, was to take full advantage of the transmitting bandwidth, avoiding the throughput limitations imposed by the physical link between host and the SDR, and being able to easily generate the transmit signal in software. Generating the transmission signal in software has the benefit that the chirp parameters can be very easily varied and that pre-distortion can be easily implemented to compensate for system non-linearities.

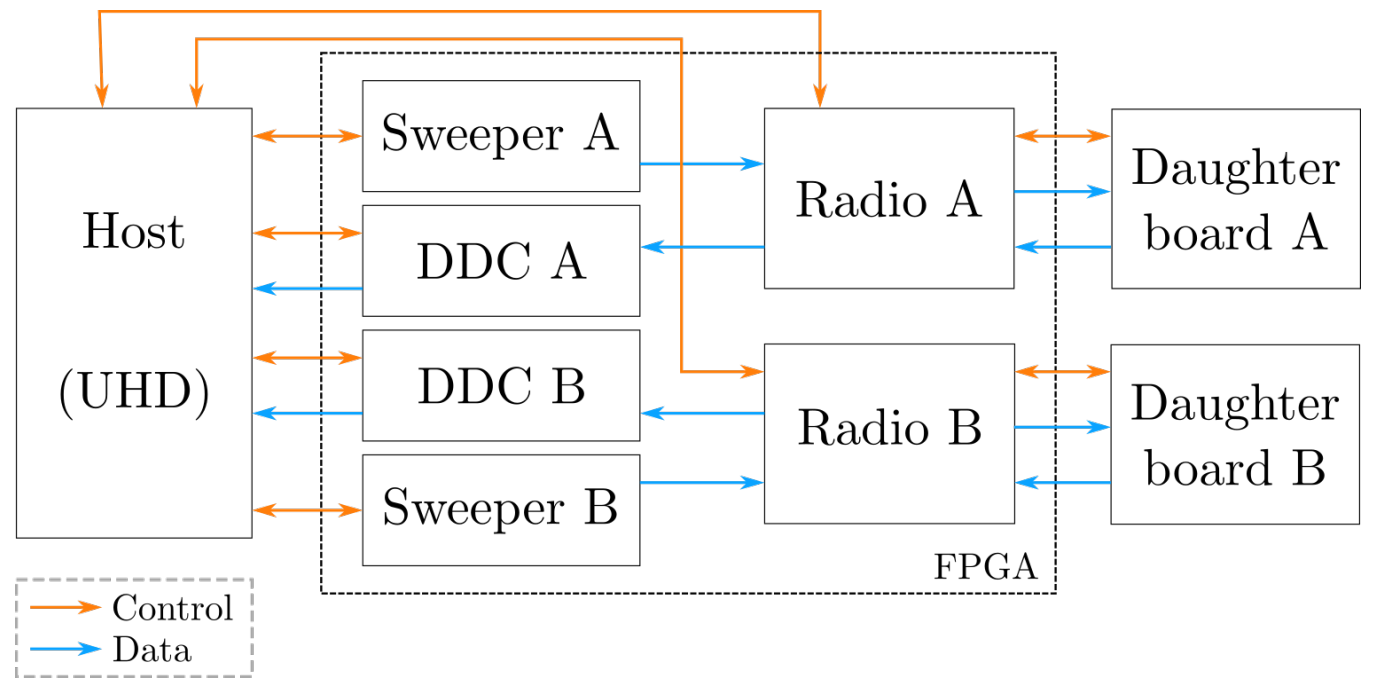

Figure 6. Simplified functional RFNoC representation of the radar core.

The radio cores interface with the ADC and the daughter-boards and are in charge of configuring the daughter-boards, managing the transmit and receive stream, and implement In-phase and Quadrature (IQ) imbalance correction through pre-distortion.

Finally, the DDC blocks perform digital down-conversion, decimation and digital frequency shifting at the same time.

\subsection{Processing Chain and Instrument Control}

Image Formation and Focusing

Once an acquisition is performed and raw data is available, there are various steps required before image formation. These steps are schematized in Figure 7a. First, the raw data samples are stored sequentially, so proper separation of individual sweeps is needed. This is done by transferring the file data into a one-dimensional array in memory and then restructuring, or reshaping it into a bi-dimensional array using the number of samples per sweep described in the metadata. The reshaping results in a bi-dimensional complex array, called the raw data array, whose dimensions are fast time samples and slow time samples. 
The raw data array contains a number of samples in slow time that greatly exceeds the Nyquist sampling requirement for image formation due to the high pulse repetition frequency (PRF) required by the operation in range. To reduce the amount of data to be stored and processed, the raw data matrix is decimated in the slow time dimension. After azimuth decimation, fast time pre-processing operations are carried out. First, a given number $N_{T F}$ of samples are trimmed from the beginning of each sweep. These samples are within the time segment corresponding to the maximum round trip delay for the given scene. Usually, during this time segment, the received signal corresponds to the last part of the previous sweep, causing a beat signal spur. The next step is to apply the one of the self-induced RFI removal algorithms explained in Section 2.3. This is a computationally expensive task and optimization is essential in order to keep image formation time acceptable. Finally, image formation is implemented through the well-known backprojection focusing algorithm [36], and a schematic view of its implementation is shown in Figure 7b. The implementation of this backprojection algorithm makes extensive use of hardware acceleration libraries such as CuPy [37], for CUDA GPU processing, and Numba [38], a just-in-time compiler that provides high performance implementations of mathematical libraries, vectorized operations and parallelization capabilities.

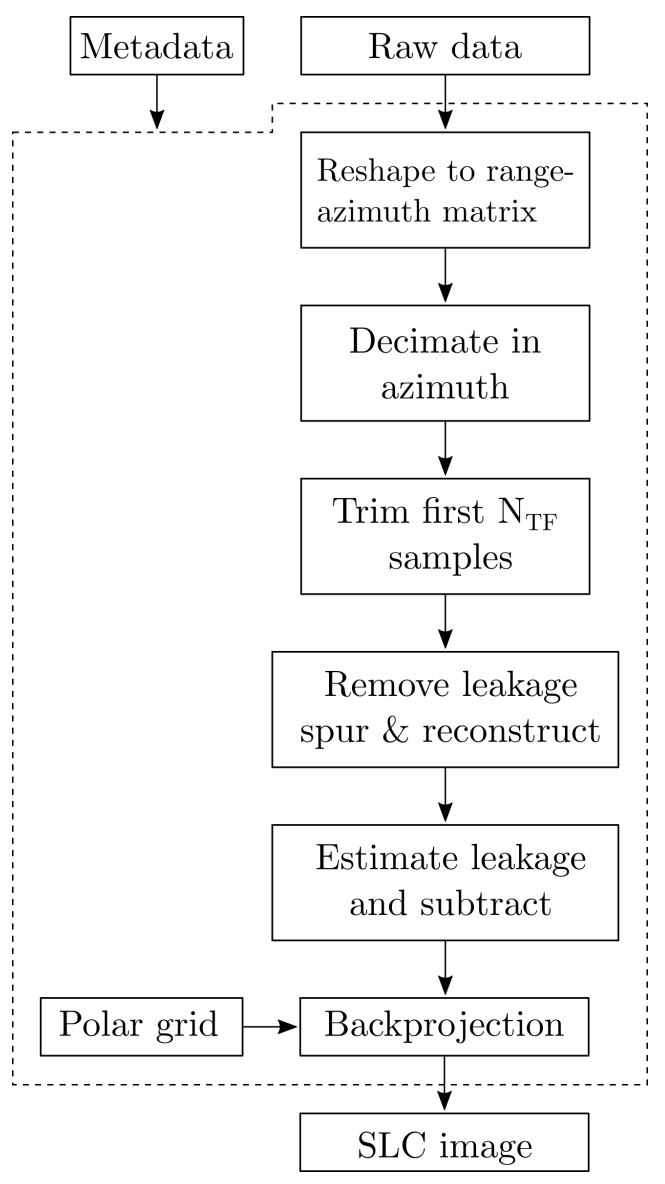

(a)

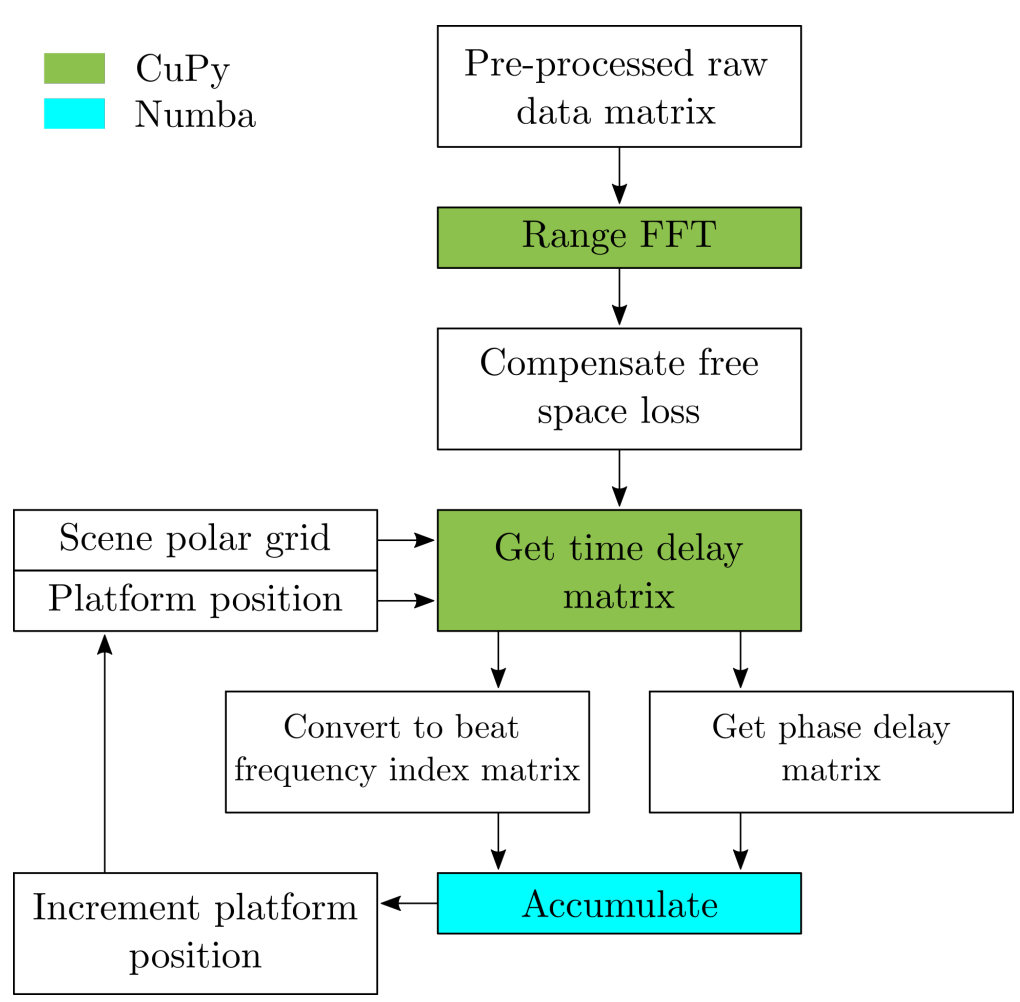

(b)

Figure 7. (a) Raw data to Single Look Complex image. (b) Schematic representation of the backprojection algorithm. Green blocks are accelerated with a GPU, while the blue block is pre-compiled and parallelized in the CPU.

\subsection{Operational Modes}

The system can operate in three basic modes. The most basic one is the real aperture mode, in which the radar unit does not move and only range discrimination can be performed. The second one is the stop-and-go mode, in which the radar unit moves along the rail stopping at each sampling position for SAR operation. The last one is the on-the-go mode, in which the radar unit does not stop and performs a continuous sampling along 
the rail. The image formation algorithms for the stop-and-go and on-the-go mode are slightly different, and each mode provides its own advantages. The stop-and-go mode is easier to process, provides more control on the total acquisition time and is able to deliver pre-averaged data, while the on-the-go mode is much less sensitive to atmospheric and de-correlation effects.

\section{Measurement Campaigns and Results}

Throughout the development of the system, extensive testing has been part of an agile design cycle. Small and progressively more complex tests have been carried out to validate different aspects of the GBSAR operation, such as signal generation, acquisition, RFI, leakage cancellation and image formation, among others. A sample of multi-frequency results is shown in this section.

\subsection{Muntanya Rodona}

One of the first test sites is located in the Muntanya Rodona area, in Subirats, south of Barcelona (Spain). It was chosen for its proximity to the company facilities, its easy accessibility and the presence of man-made targets such as buildings and other urban structures, which allowed for a fast assessment of correct synthetic image formation. The results presented here represent the two last iterations performed on this site.

The main objective pursued in this test campaign was to reach a system maturity point that ensured its robust operation in future campaigns. This objective can be divided in smaller milestones:

- To ensure the reliable operation of the RF front-end, SDR and the linear unit.

- To automate most software procedures and system configuration, while ensuring the reliability of the software.

- To test the on-the-go acquisition mode.

- To perform a qualitative assessment of the characteristics of measurements done at all frequencies.

In all the cases, the radar was located at $41^{\circ} 22^{\prime} 41.6^{\prime \prime} \mathrm{N} 1^{\circ} 49^{\prime} 00.7^{\prime \prime} \mathrm{E}$; see the urban area as shown in Figure $8 \mathrm{~b}$. The urban area is on top of a small hill, with a maximum approximate elevation of $36 \mathrm{~m}$ with respect to the radar location as can be seen in Figure $8 \mathrm{a}$. The targets imaged lie within a range from $100 \mathrm{~m}$ to $200 \mathrm{~m}$. The scene can be seen from the radar point of view in Figure 8c. A small corner reflector was placed at $100 \mathrm{~m}$ range, approximately. Although stop-and-go and on-the-go acquisitions were performed in the first measurements, all results presented from now on were acquired using the on-the-go mode. The reason for this is that once the on-the-go mode processing was operational, the stop-and-go mode was deemed inferior in all aspects, the acquisition time being the worst one in practical terms.

The logarithmic power Single Look Complex (SLC) images can be seen in Figure 9. From these, a qualitative assessment can be done about the characteristics of each band. The first effect to note is the evident loss of resolution, which worsens progressively as the frequency decreases. At X-and C-bands, the resolution is high enough to be able to correlate the orthophoto from Figure $8 \mathrm{~b}$ with the radar images, the buildings and the staggered geometry of the terrain being the most distinctive features. At L-band, this correlation is more difficult to observe, while at P-band the shape of the scene is lost. The second effect to note is the progressive broadening of the illuminated scene, consistent with the broadening of the antenna beam patterns shown in Figure $4 \mathrm{~b}-\mathrm{d}$. Another relevant aspect is the superior vegetation penetration characteristics in $\mathrm{L}$ and P-bands, as can be seen, for example, at coordinates $(170,-70)$. Additionally, a noticeable aspect is the dimming of the reflector as the frequency decreases, an effect of the decrease of the reflector radar cross section (RCS), combined with its smaller contribution within a bigger pixel. 


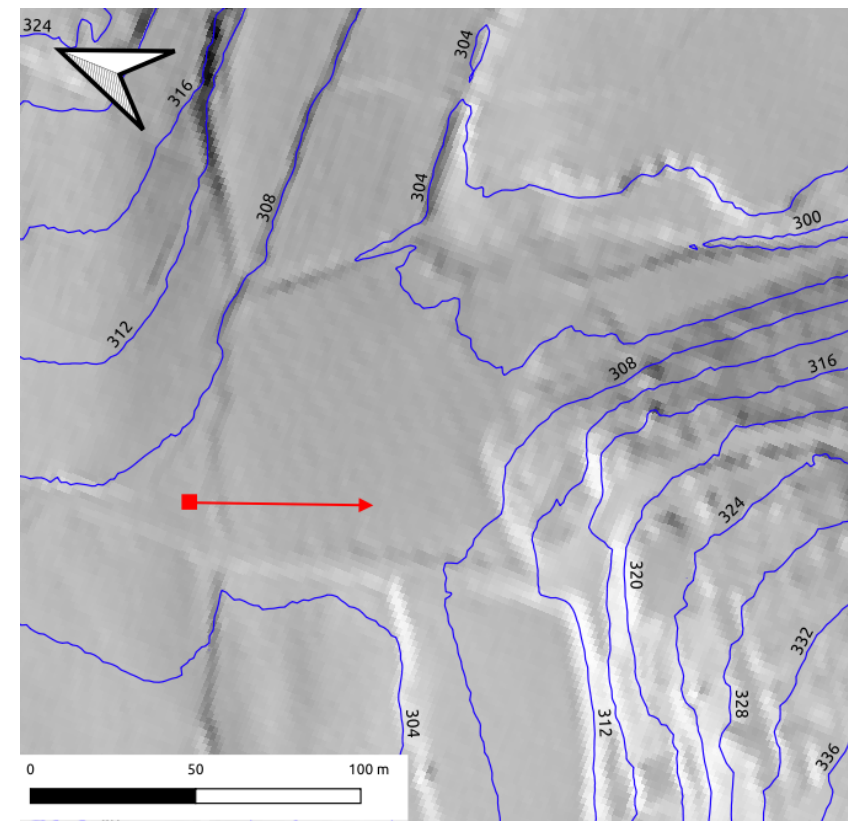

(a)

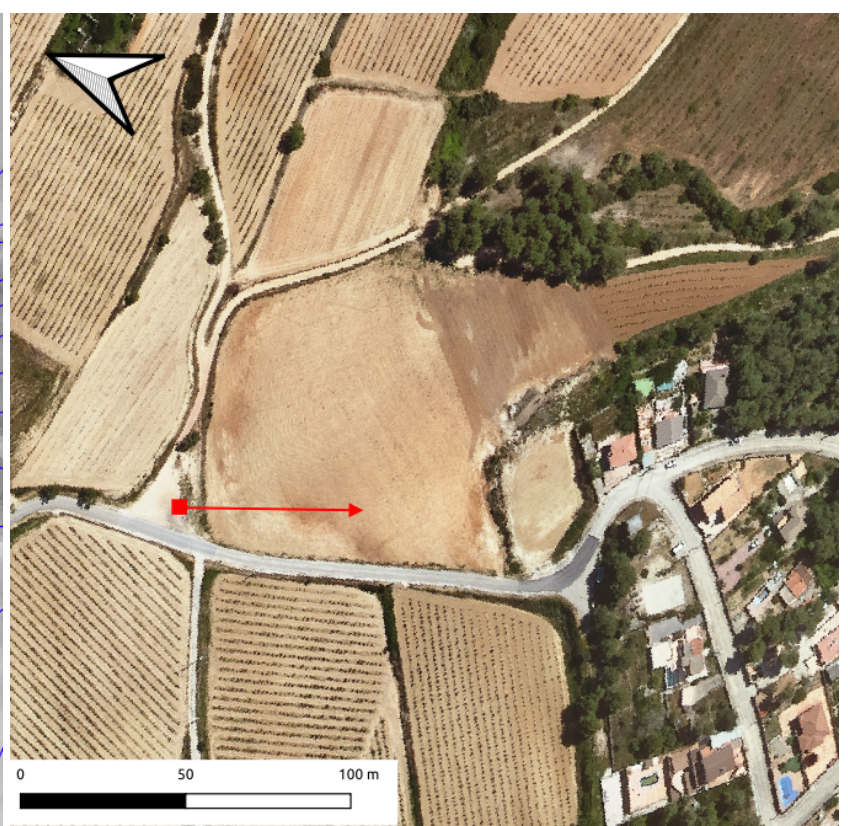

(b)

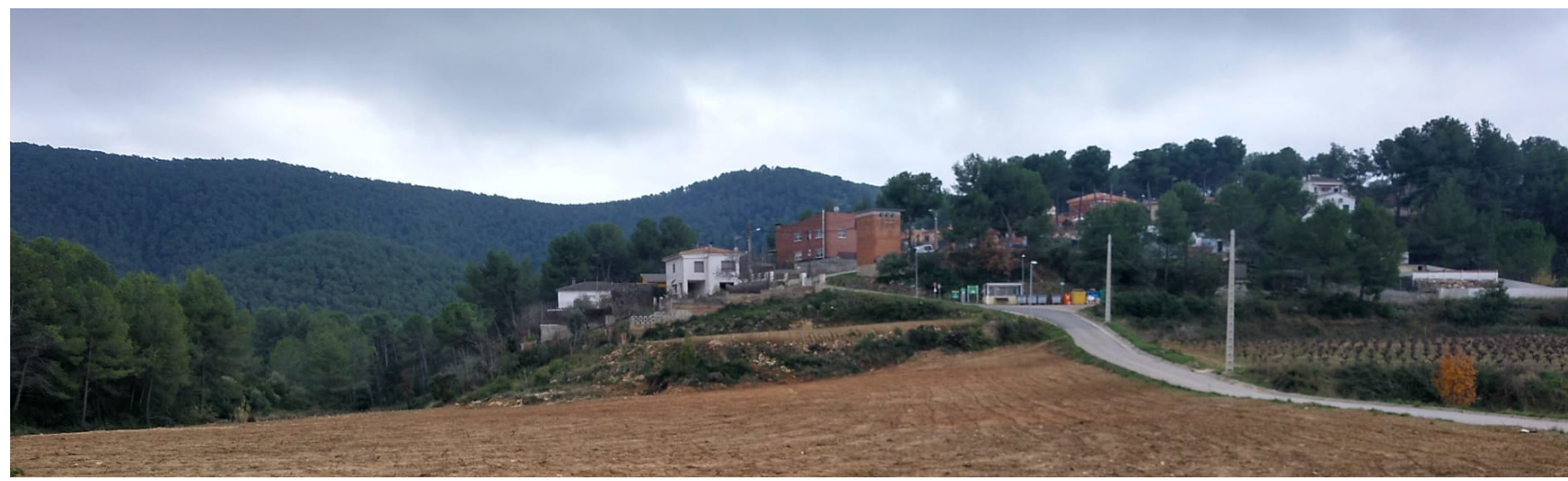

(c)

Figure 8. Muntanya Rodona test area maps. (a) Elevation map. (b) Orthophoto. (c) Muntanya Rodona test area view from the radar position. The red square and arrow specify the radar location and azimuth look direction. Orthophoto and DEM credit: Institut Cartogràfic i Geològic de Catalunya.

\subsection{Castell de Subirats}

The second batch of tests was done in the Subirats Castle in Subirats, south of Barcelona (Spain). These tests were aimed at performing more advanced tests that consisted on the generation of multi-frequency images of big areas.

The northern view from the Subirats castle is diverse. A speedway, various roads, industrial buildings and a high speed train bridge can be seen, combined with vineyards and other vegetated zones. The radar was located at $41^{\circ} 24^{\prime} 59.0^{\prime \prime} \mathrm{N} 1^{\circ} 48^{\prime} 59.5^{\prime \prime} \mathrm{E}$, at an altitude of $285 \mathrm{~m}$ above sea level. As a reference, the height difference between the radar and the speedway is $146 \mathrm{~m}$. An orthophoto and its corresponding elevation map are shown in Figure 10. The radar was configured to image an area between $200 \mathrm{~m}$ and $2 \mathrm{~km}$. Only two images were taken in each band. The objectives of this test were the same as at the second test site, but in this case the imaged area was more diverse. In Figure 11 the area from the radar point of view is shown, along with a detail of some vineyards and artificial structures. To assist in the comprehension of the geometry of the area, height and slope profiles are shown in Figure 12. 

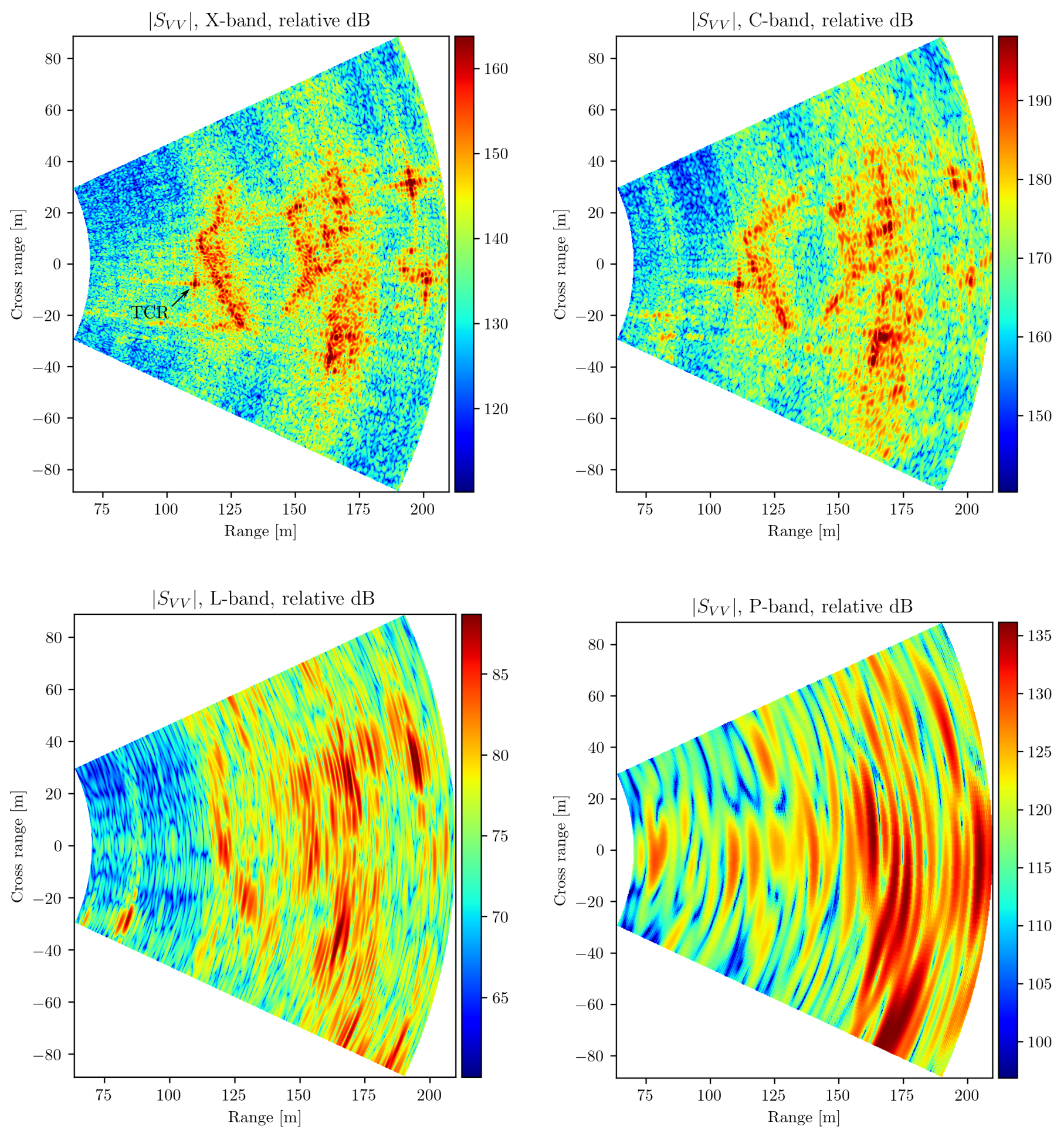

Figure 9. SLC power in decibels at all four frequencies. The absolute power is uncalibrated. 


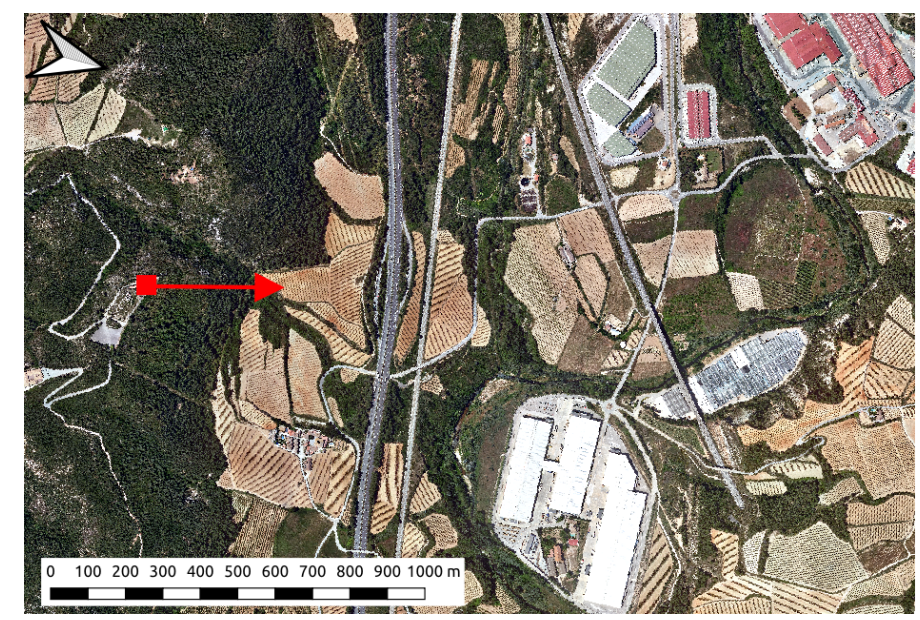

(a)

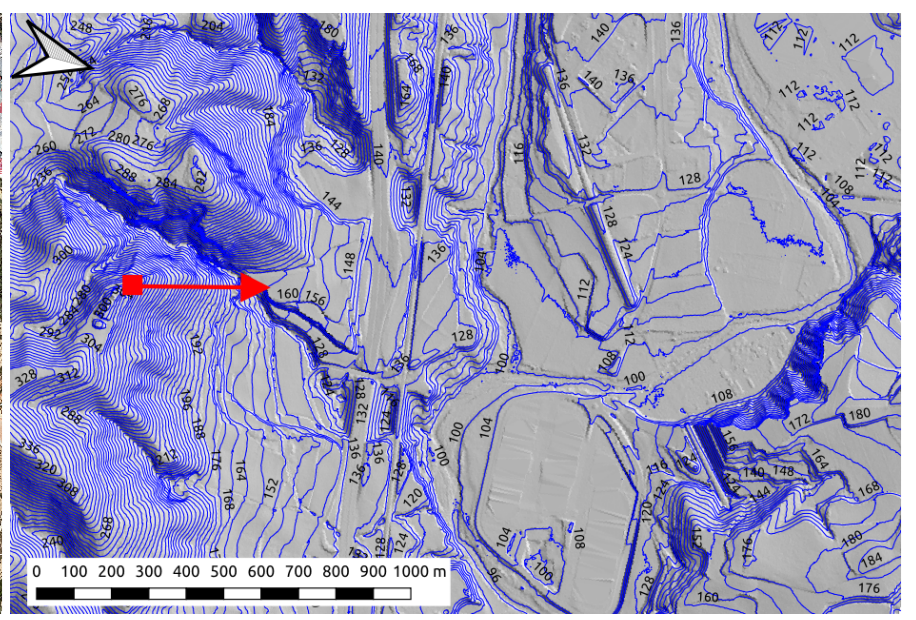

(b)

Figure 10. Test site $\mathrm{n}^{\circ} 3$ orthophoto (a) and elevation map (b). The square and arrow indicate the radar position and azimuth view direction. Orthophoto and DEM credit: Institut Cartogràfic i Geològic de Catalunya.

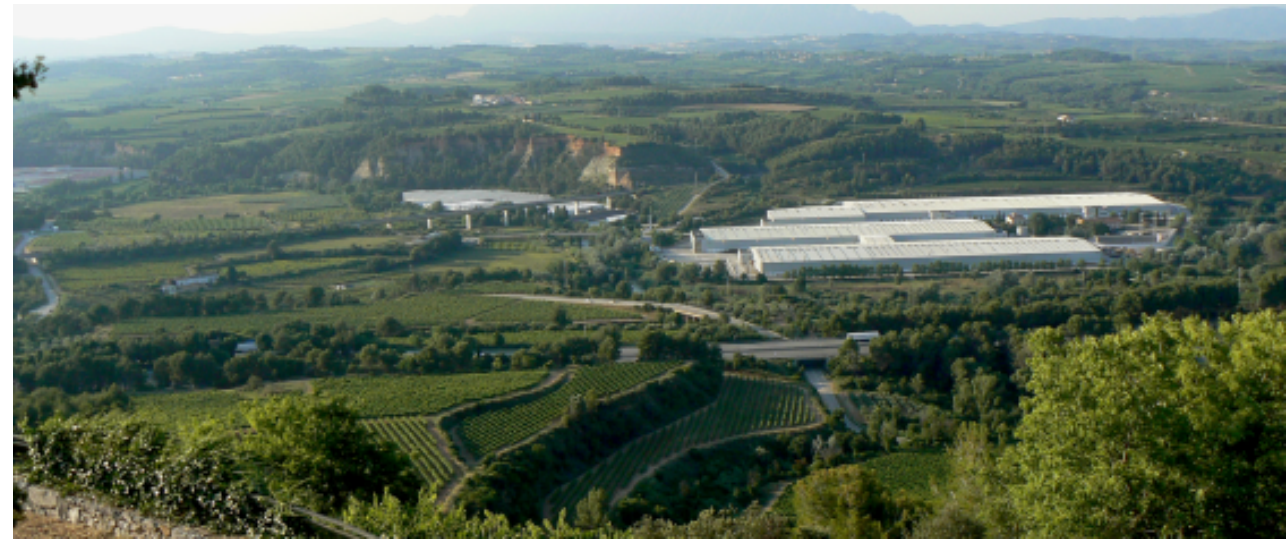

(a)

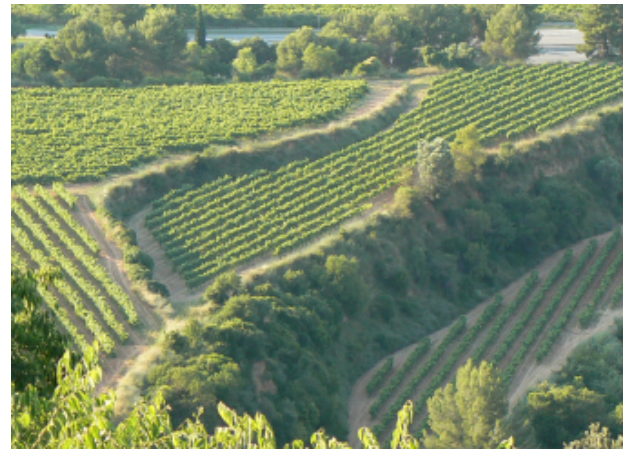

(b)

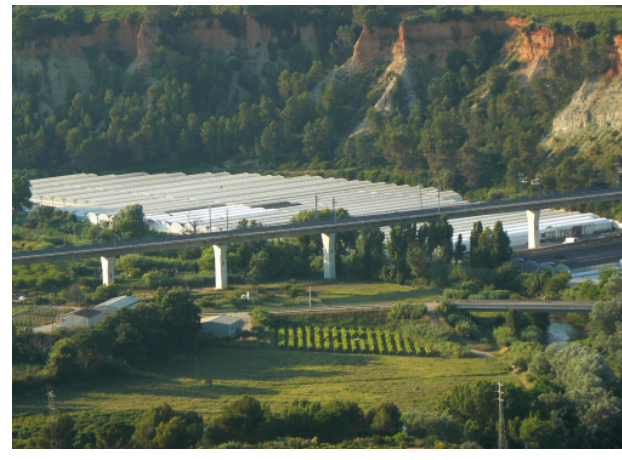

(c)

Figure 11. (a) Test site $n^{\circ} 3$ from the radar point of view. (b) Vineyard area in detail. (c) A detailed view of the area around the high-speed train bridge. 


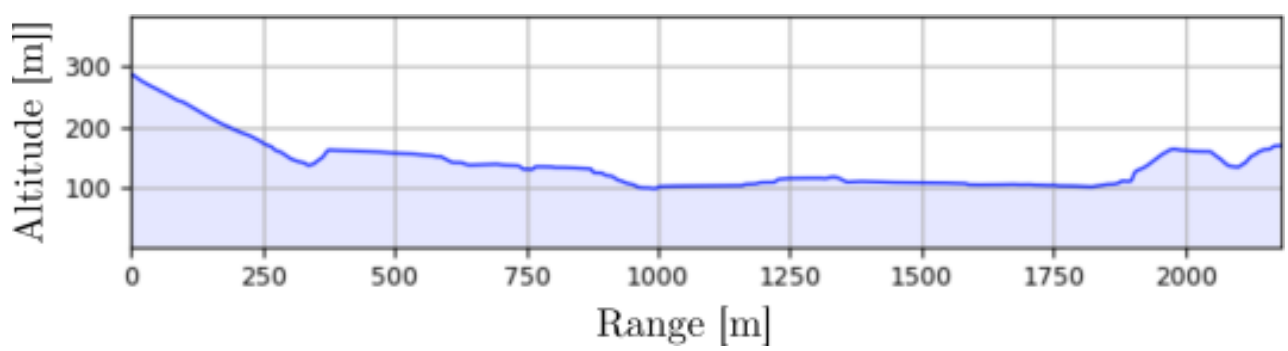

Figure 12. Height profile in the zero azimuth direction. The horizontal axis represents range. All dimensions are in meters. DEM credit: Institut Cartogràfic i Geològic de Catalunya.

The magnitude images are shown in Figure 13, and the accompanying X-band thresholded magnitude composite image is shown in Figure 14 for reference. Unexpectedly, a spur at zero azimuth is present in the focused images, which was not present in the near range ones. This spur was determined to be related with insufficient isolation between the front-end reference clock and the RF signals. It is only visible at far ranges due to its high power with respect to the backscattered power from the scene. An isolation improvement was implemented in the subsequent front-end revision. In the images shown here, the spur was removed through the removal and interpolation of the DC bin in the slow time Fourier domain. When inspecting the X-band image, a good correlation can be seen between the magnitude and the characteristics of the area. From low to high , the vineyard appears relatively strongly (Figure 15a), followed by various smaller artificial reflectors, such as metallic bridge railings (Figure 15b) and parked vehicles in a motorway rest area. Starting at $1 \mathrm{~km}$, the strongest reflectors in the image can be seen to have originated from the metallic shapes present in industrial buildings (Figure 15c). Advancing in range, other artificial buildings and structures such as bridges and electrical towers result in strong returns (Figure 15d), and the train bridge is also clearly seen.
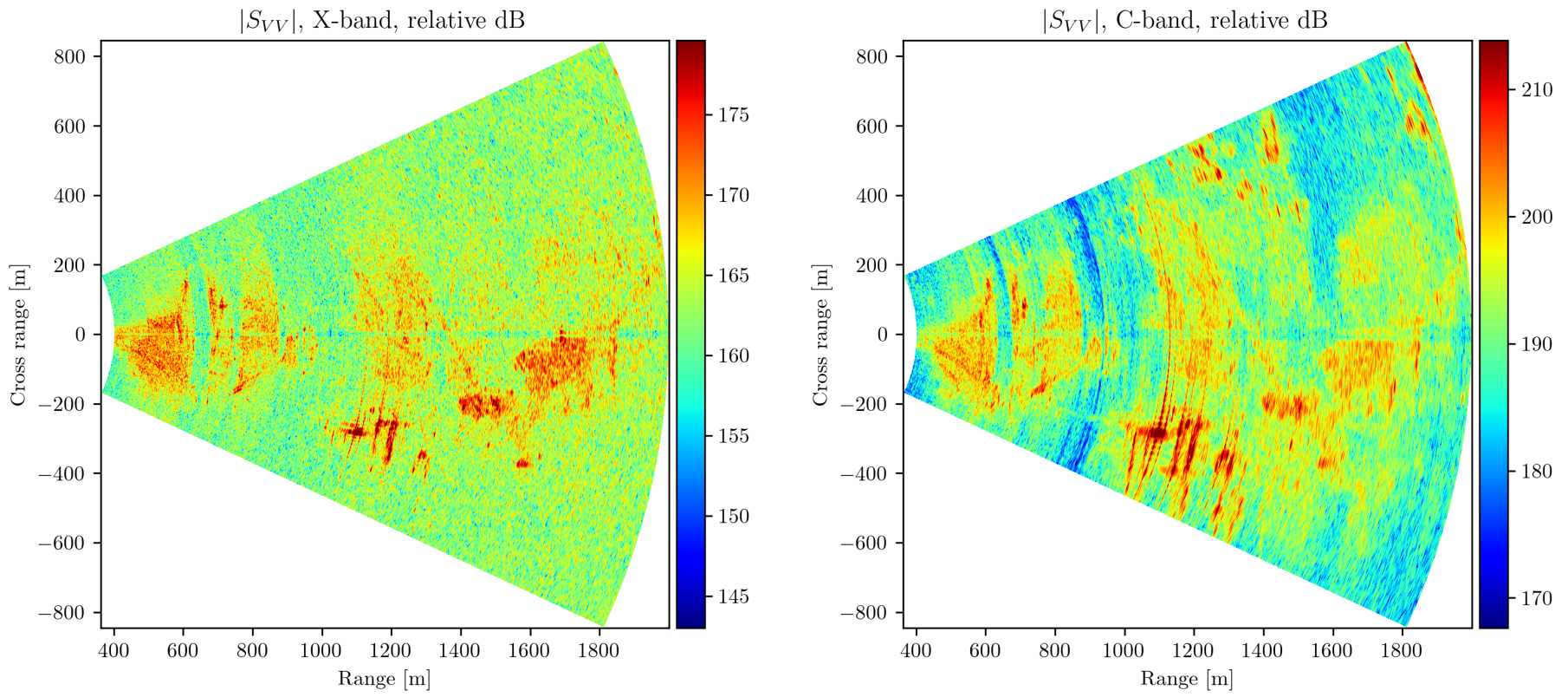

Figure 13. Cont. 

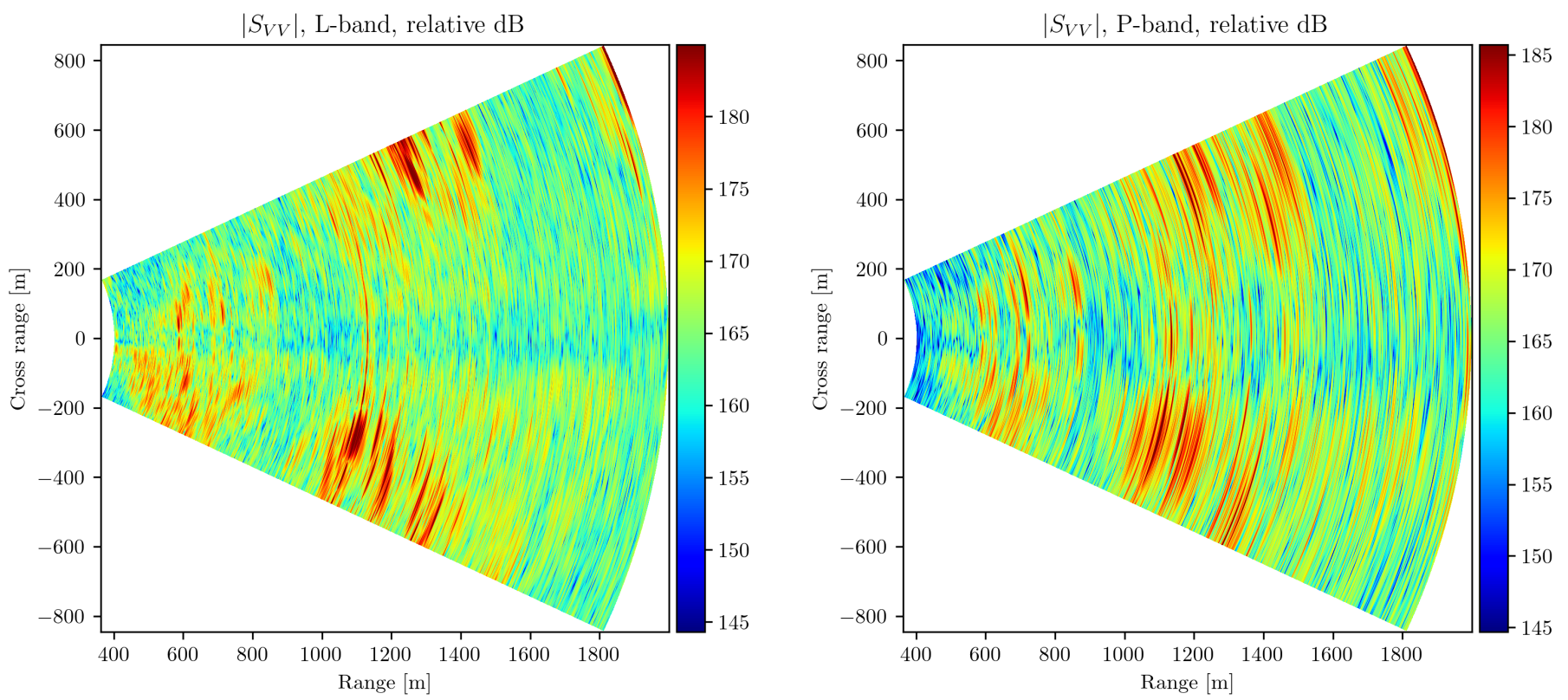

Figure 13. Magnitude images of the test site $n^{\circ} 3$. The absolute power is uncalibrated.

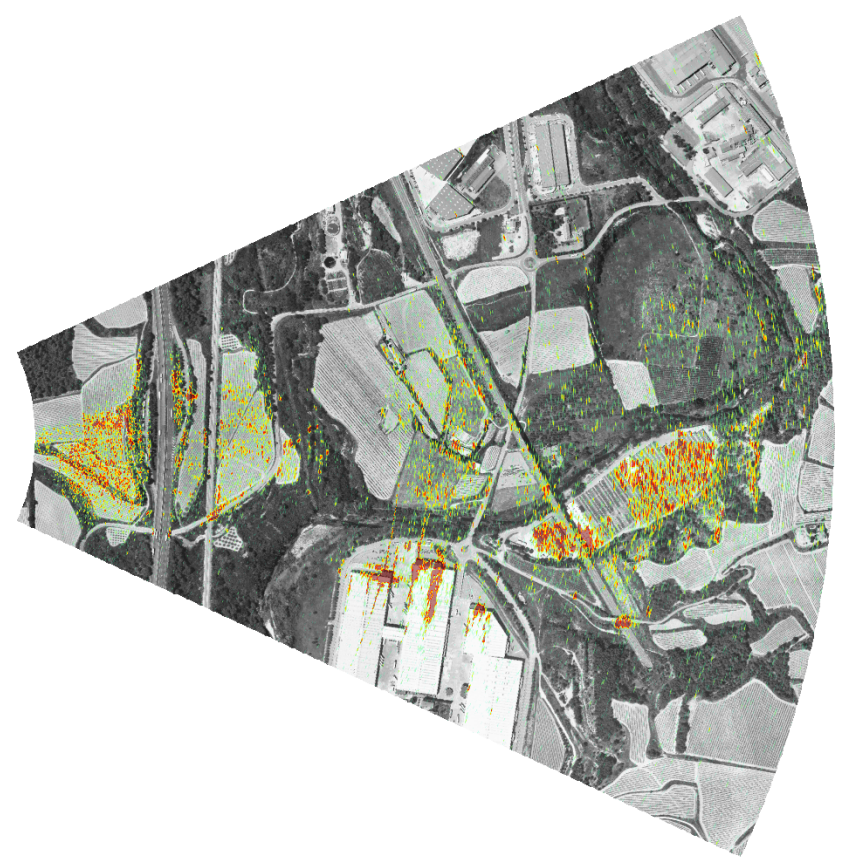

Figure 14. Composition of the orthophoto and the thresholded magnitude X-band GBSAR image of the Subirats castle test site $n^{\circ} 3$. Orthophoto credit: Institut Cartogràfic i Geològic de Catalunya.

In C-band the same effect observed in the test site $\mathrm{n}^{\circ} 2$ took place, including the appearance of targets at high azimuth angles due to the broadening of the antenna beam, and an increase in the dynamic range resulted from significantly less free-space loss. In Lband, the degradation in resolution and the central spur made interpretation more difficult. An interesting observation is that the artificial structures around $(1700,-100) \mathrm{m}$ yielded a much lower return relative to the rest of the image, indicating that the contributing reflectors were small compared with the L-band wavelength. For P-band the same observation can be made. 


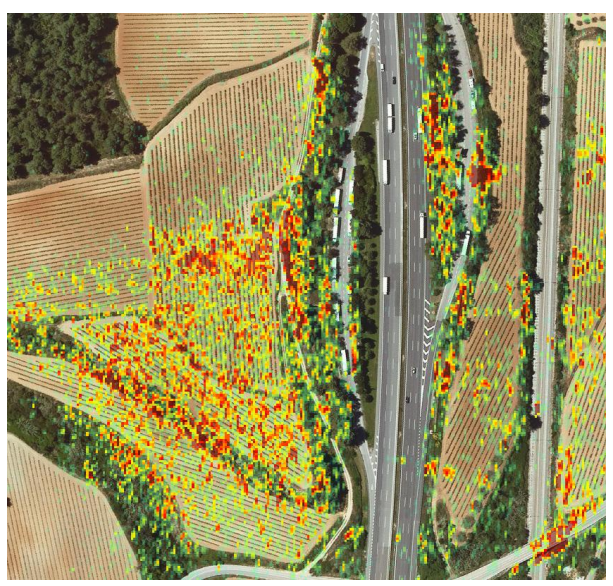

(a)

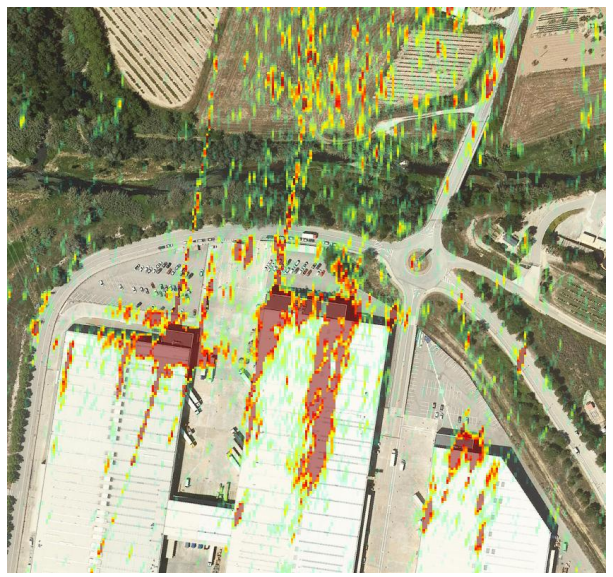

(c)

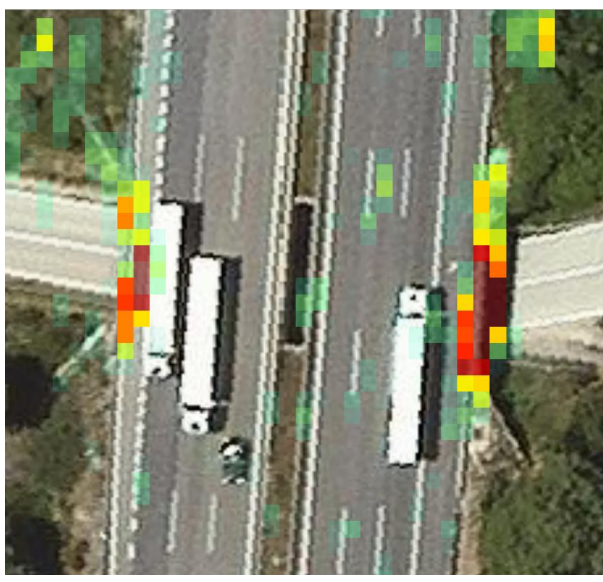

(b)

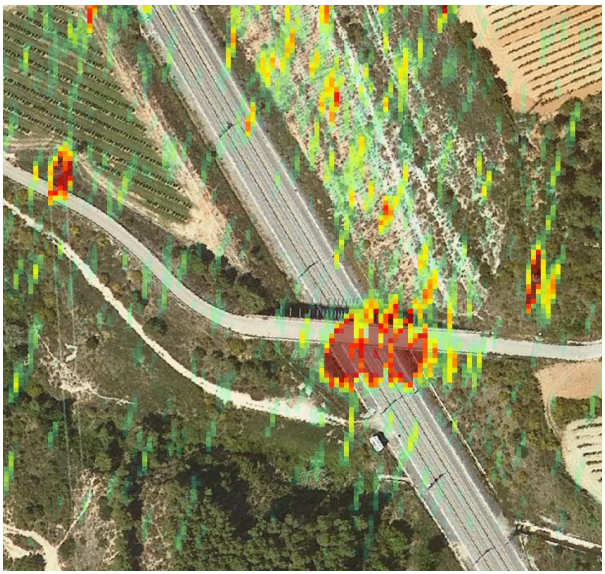

(d)

Figure 15. Details of the thresholded linear magnitude image for test site $n^{\circ} 3$. Optical images were not taken at the same time. (a) vineyard (b) metallic bridge railings (c) industrial buildings (d) other artificial buildings and structures.

\section{Conclusions}

The main contribution of this work lies in the demonstration that the most common SDR architecture can be exploited for the implementation of high performance radars, especially a GBSAR. Differently from other works, this work specifically demonstrated the implementation of a GBSAR comparable in performance to other commercial systems. This was possible by working on two fundamental problems observed in previous works: the limited processing performance of the host device, which limits the range resolution, and the spurious content of Zero-IF transceivers, which prevented the generation of fullbandwidth chirps. A signal generation block was implemented in the FPGA, taking full advantage of the available signal bandwidth. Another relevant contribution was the usage of signal reconstruction algorithms to circumvent the effects of the SDR transceiver, once again pursuing the objective of using all the available RF bandwidth.

The multi-frequency GBSAR system opens up new possibilities. One of them, for instance, is the selection of a frequency band depending on the strength of decorrelation effects, such as the movement of vegetation or scatterers, such as small rocks, that yield low interferometric coherence values, and hence, low-quality data. In the case of differential interferometry, the multi-frequency capability would allow one to select a low-frequency band when the terrain movement causes severe phase wrapping problems, or to even use a low-frequency band to assist in the phase unwrapping process of a high-frequency image. Another potential application is the usage of data fusion algorithms that fuse together the information of multiple bands to yield richer datasets. For instance, areas that exhibit high 
coherence at the high frequencies can be sensed with the high-frequency bands, exploiting the high azimuth resolution; and areas exhibiting low coherence can be sensed instead with low-frequency bands that—even with low resolutions-can provide valuable information. Other potential applications are the ones that arise from the combination of multi-frequency and hyper-temporal (acquisitions densely spaced in time) imagery.

Author Contributions: C.L.-M. conceived the project; A.A. designed and built the instrument; A.A. designed and implemented the software; C.L.-M. and R.J. acquired the funding; C.L.-M. provided scientific support; A.A. and R.J. participated in the field tests; A.A. wrote the paper. All authors have read and agreed to the published version of the manuscript.

Funding: This work was funded by the Spanish Ministry of Economy and Competitiveness through grant number DI-15-08147; a grant to "CommSensLab-UPC" Excellence Research Unit Maria de Maeztu, grant number MDM-2016-600; the Catalan Ministry of Business and Knowledge, grant number 2016 DI 012; and a Spanish Ministry of Science, Innovation and Universities project, grant number RTI2018-099008-B-C21, "Sensing with pioneering opportunistic techniques."

Institutional Review Board Statement: Not applicable.

Informed Consent Statement: Not applicable.

Data Availability Statement: The data generated for this manuscript is not publicly available.

Acknowledgments: The authors want to acknowledge the technical support given by Ricard Gonzálvez and the scientific support and encouragement of Adriano Camps.

Conflicts of Interest: The authors declare no conflict of interest. The funders had no role in the design of the study; in the collection, analyses, or interpretation of data; in the writing of the manuscript, or in the decision to publish the results.

$\begin{array}{ll}\text { Abbreviations } \\ \text { The following abbreviations are used in this manuscrip } \\ \text { GBSAR } & \text { Ground-Based Synthetic Aperture Radar } \\ \text { SDR } & \text { Software Defined Radio } \\ \text { FMCW } & \text { Frequency-Modulated Continuous Wave } \\ \text { UHD } & \text { USRP Hardware Driver } \\ \text { SAR } & \text { Synthetic Aperture Radar } \\ \text { IQ } & \text { In-phase and Quadrature } \\ \text { IF } & \text { Intermediate Frequency } \\ \text { C } & \text { RF Network-on-Chip } \\ \text { PRF } & \text { Pulse Repetition Frequency } \\ \text { FPGA } & \text { Field Programmable Gate Array } \\ \text { VNA } & \text { Vector Network Analyzer } \\ \text { PRF } & \text { Pulse Repetition Frequency } \\ \text { RAM } & \text { Random Access Memory }\end{array}$

\section{References}

1. IEEE Standard for Definitions and Concepts for Dynamic Spectrum Access: Terminology Relating to Emerging Wireless Networks, System Functionality, and Spectrum Management; IEEE Std 1900.1-2019 (Revision of IEEE Std 1900.1-2008); Wiley-IEEE Press: New York, NY, USA, 2019; pp. 1-78. [CrossRef]

2. Wiesbeck, W. SDRS: Software-defined radar sensors. In Proceedings of the IGARSS 2001, Scanning the Present and Resolving the Future, Proceedings, IEEE 2001 International Geoscience and Remote Sensing Symposium (Cat. No.01CH37217), Sydney, NSW, Australia, 9-13 July 2001; Volume 7, pp. 3259-3261. [CrossRef]

3. Patton, L.K., A GNU Radio Based Software-Defined Radar. Master's Thesis, Wright State University, Dayton, OH, USA, 2007.

4. Rossler, C.W.; Ertin, E.; Moses, R.L. A software defined radar system for joint communication and sensing. In Proceedings of the IEEE National Radar Conference, Kansas City, MO, USA, 23-27 May 2011; pp. 1050-1055. [CrossRef]

5. Costanzo, S.; Spadafora, F.; Borgia, A.; Moreno, O.; Costanzo, A.; Di Massa, G.; Moreno, H.O.; Costanzo, A.; Di Massa, G. High resolution software defined radar system for target detection. Adv. Intell. Syst. Comput. 2013, 206. [CrossRef]

6. Spadafora, F.; Massa, G.D.; Borgia, A.; Costanzo, A.; Aloi, G.; Pace, P.; Loscr, V. Potentialities of Usrp-Based Software Defined Radar Systems. Prog. Electromagn. Res. B 2013, 53, 417-435. 
7. Hershberger, J.; Pratt, T.; Kossler, R. A software-defined, dual-polarized radar system. In Proceedings of the 2016 IEEE Conference on Antenna Measurements and Applications (CAMA), Syracuse, NY, USA, 23-27 October 2016; pp. 1-4. [CrossRef]

8. Petri, D.; Berizzi, F.; Martorella, M.; Dalle Mese, E.; Capria, A. A software defined UMTS passive radar demonstrator. In Proceedings of the 4th Microwave and Radar Week MRW-2010-11th International Radar Symposium, IRS 2010-Conference Proceedings, Vilnius, Lithuania, 16-18 June 2010; pp. 44-47.

9. Heunis, S.; Paichard, Y.; Inggs, M. Passive Radar using a Software-Defined Radio Platform and Opensource Software Tools. In Proceedings of the IEEE RadarCon (RADAR), Kansas City, MO, USA, 23-27 May 2011; pp. 879-884. [CrossRef]

10. Beadle, E.R.; Dyson, T. Software-Based Reconfigurable Computing Platform ( AppSTAR TM ) for Multi-Mission Payloads in Spaceborne and Near-Space Vehicles. In Proceedings of the International Conference on Reconfigurable Systems and Algorithms, Las Vegas, NV, USA, 16-19 July 2012; pp. 25-31.

11. Pancik, J.; Pancik, M. Hardware and software front-end based on the USRP for experimental X-band Synthetic Aperture Radar. In Proceedings of the 25th International Conference Radioelektronika, RADIOELEKTRONIKA 2015, Pardubice, Czech Republic, 21-22 April 2015; pp. 154-159. [CrossRef]

12. Grabowski, A. SDR-based LFM signal generator for radar/SAR systems. In Proceedings of the 2016 17th International Radar Symposium (IRS), Krakow, Poland, 10-12 May 2016; pp. 1-3. [CrossRef]

13. Liu, P.; Mendoza, J.; Hu, H.; Burkett, P.G.; Urbina, J.V.; Anandakrishnan, S.; Bilén, S.G. Software-Defined Radar Systems for Polar Ice-Sheet Research. IEEE J. Sel. Top. Appl. Earth Obs. Remote Sens. 2019, 12, 803. [CrossRef]

14. Frankford, M.T.; Majurec, N.; Johnson, J.T. Software-defined radar for MIMO and adaptive waveform applications. In Proceedings of the IEEE National Radar Conference, Arlington, VA, USA, 10-14 May 2010; pp. 724-728. [CrossRef]

15. Mealey, T.C.; Duly, A.J. BEEMER: A firmware-tuned, software-defined MIMO radar testbed. In Proceedings of the IEEE International Symposium on Phased Array Systems and Technology, Waltham, MA, USA, 18-21 October 2016. [CrossRef]

16. Bell, K.L.; Johnson, J.T.; Smith, G.E.; Baker, C.J.; Rangaswamy, M. Cognitive radar for target tracking using a software defined radar system. In Proceedings of the 2015 IEEE Radar Conference (RadarCon), Arlington, VA, USA, 10-15 May 2015; pp. 1394-1399. [CrossRef]

17. Kirk, B.H.; Owen, J.W.; Narayanan, R.M.; Blunt, S.D.; Martone, A.F.; Sherbondy, K.D. Cognitive software defined radar: waveform design for clutter and interference suppression. Radar Sens. Technol. XXI 2017, 10188, 1018818. [CrossRef]

18. Christiansen, J.M.; Smith, G.E.; Olsen, K.E. USRP based cognitive radar testbed. In Proceedings of the 2017 IEEE Radar Conference (RadarConf), Seattle, WA, USA, 8-12 May 2017; pp. 1115-1118. [CrossRef]

19. Pieraccini, M.; Miccinesi, L. Ground-based radar interferometry: A bibliographic review. Remote Sens. 2019, 11, 1029. [CrossRef]

20. Monserrat, O.; Crosetto, M.; Luzi, G. A review of ground-based SAR interferometry for deformation measurement. ISPRS J. Photogramm. Remote Sens. 2014, 93, 40-48. [CrossRef]

21. Caduff, R.; Schlunegger, F.; Kos, A.; Wiesmann, A. A review of terrestrial radar interferometry for measuring surface change in the geosciences. Earth Surf. Process. Landf. 2015, 40, 208-228. [CrossRef]

22. Papathanassiou, K.P.; Moreira, J.R. Interferometric analysis of multifrequency and multipolarization SAR data. In Proceedings of the International Geoscience and Remote Sensing Symposium (IGARSS), Lincoln, NE, USA, 31 May 1996; Volume 2, pp. 1227-1229. [CrossRef]

23. Wahl, M.; Ottl, H.; Velten, E. Programme aspects for future multifrequency/multipolarization SAR. In Proceedings of the 1995 International Geoscience and Remote Sensing Symposium, IGARSS '95, Quantitative Remote Sensing for Science and Applications, Firenze, Italy, 10-14 July 1995; Volume 3, pp. 1834-1835. [CrossRef]

24. Baselice, F.; Ferraioli, G.; Reale, D. Multi-frequency and multi-baseline ground based SAR for environmental monitoring. In Proceedings of the 2015 IEEE International Workshop on Measurements and Networking, M and N 2015-Proceedings, Coimbra, Portugal, 12-13 October 2015; pp. 123-128. [CrossRef]

25. Farina, A.; Morabito, F.C.; Serpico, S.; Simone, G. Fusion of radar images: State of art and perspective. In Proceedings of the CIE International Conference of Radar Proceedings, Beijing, China, 15-18 October 2001; pp. 9-15. [CrossRef]

26. Martinez-Vazquez, A.; Fortuny-Guasch, J.; Gruber, U. Monitoring of the snow cover with a ground-based synthetic aperture radar. EARSeL eProc. 2005, 4, 171-178.

27. Navarro-Sanchez, V.D.; Lopez-Sanchez, J.M.; Ferro-Famil, L. Polarimetric approaches for persistent scatterers interferometry. IEEE Trans. Geosci. Remote Sens. 2014, 52, 1667-1676. [CrossRef]

28. Mougin, E.; Proisy, C.; Marty, G.; Fromard, F.; Puig, H.; Betoulle, J.L.; Rudant, J.P. Multifrequency and multipolarization radar backscattering from mangrove forests. IEEE Trans. Geosci. Remote Sens. 1999, 37, 94-102. [CrossRef]

29. IDS Ingegneria Dei Sistemi S.p.A. IBIS-FM IDS GeoRadar Website. Available online: https://idsgeoradar.com/products/ interferometric-radar/ibis-fm (accessed on 20 April 2021).

30. Metasensing BV. FastGBSAR-S I Geomatics Website. Available online: https://www.geomatics.metasensing.com/fastgbsar-s (accessed on 20 April 2021).

31. Barton, D.K. Modern Radar System Analysis; Artech House Radar Library: Norwood, MA, USA, 1988.

32. Edwards, M.; Madsen, D.; Stringham, C.; Margulis, A.; Wicks, B.; Long, D.G. MicroASAR: A small, robust LFM-CW SAR for operation on UAVS and small aircraft. In Proceedings of the International Geoscience and Remote Sensing Symposium (IGARSS), Boston, MA, USA, 7-11 July 2008; Volume 5, pp. 514-517. [CrossRef] 
33. Vallant, G.; Epp, M.; Schlecker, W.; Schneider, U.; Anttila, L.; Valkama, M. Analog IQ impairments in Zero-IF radar receivers: Analysis, measurements and digital compensation. In Proceedings of the 2012 IEEE I2MTC_International Instrumentation and Measurement Technology Conference, Proceedings, Graz, Austria, 13-16 May 2012; pp. 1703-1707. [CrossRef]

34. Neemat, S.; Krasnov, O.; Yarovoy, A. An interference mitigation technique for FMCW radar using beat-frequencies interpolation in the STFT domain. IEEE Trans. Microw. Theory Tech. 2019, 67, 1207-1220. [CrossRef]

35. Prager, S.; Thrivikraman, T.; Haynes, M.; Stang, J.; Hawkins, D.; Moghaddam, M. Ultra-wideband synthesis for high-range resolution software defined radar. In Proceedings of the 2018 IEEE Radar Conference, RadarConf 2018, Oklahoma City, OK, USA, 23-27 April 2018; pp. 1089-1094. [CrossRef]

36. Soumekh, M. Synthetic Aperture Radar Signal Processing with MATLAB Algorithms; Wiley-Interscience: New York, NY, USA, 1999.

37. CuPy: A NumPy-Compatible Array Library Accelerated by CUDA. Available online: https://cupy.dev/ (accessed on 20 April 2021).

38. Numba: A High Performance Python Compiler. Available online: http://numba.pydata.org/ (accessed on 20 April 2021). 